\title{
Originalien
}

Gefässchirurgie $2020 \cdot 25: 568-574$ https://doi.org/10.1007/s00772-020-00681-w Online publiziert: 24. August 2020

(c) Der/die Autor(en) 2020

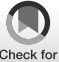

T. Schmitz-Rixen ${ }^{1} \cdot$ M. Steffen ${ }^{2}$ D. Böckler ${ }^{3} \cdot$ R. T. Grundmann ${ }^{4,5}$

${ }^{1}$ Klinik für Gefäß- und Endovascularchirurgie und des Universitären Wundzentrums, Klinikum der GoetheUniversität, Frankfurt/M, Deutschland

${ }^{2}$ Klinikum Saarbrücken, Saarbrücken, Deutschland

${ }^{3}$ Klinik für Gefäßchirurgie und Endovaskuläre Chirurgie, Universitätsklinikum Heidelberg, Heidelberg, Deutschland

${ }^{4}$ Universitäres Herz- und Gefäßzentrum UKE Hamburg, Universitätsklinikum Hamburg-Eppendorf, Hamburg, Deutschland

${ }^{5}$ Deutsches Institut für Gefäßmedizinische Gesundheitsforschung (DIGG) der Deutschen Gesellschaft für Gefäßchirurgie und Gefäßmedizin, Berlin, Deutschland

\section{Offene und endovaskuläre Versorgung des infrarenalen vs. juxtarenalen Bauchaortenaneurysmas}

\section{Patienten und Methodik}

anhand einer Registererhebung des Deutschen Instituts für Gefäßmedizinische Gesundheitsforschung (DIGG) der Deutschen Gesellschaft für Gefäßchirurgie und Gefäßmedizin (DGG) der Frage nach, ob sich die perioperativen Ergebnisse bei elektiver Versorgung juxtarenaler abdomineller Aortenaneurysmen (AAA) von denen bei Versorgung infrarenaler AAA unterscheiden. Dabei wurden sowohl offen (OAR) als auch endovaskulär (EVAR) behandelte Patienten eingeschlossen. Eine solche Auswertung liegt bisher nicht vor. In allen früheren Jahresberichten zum AAA-Register des DIGG, so auch in dem für das Jahr 2018 [14], sind stets infra- und juxtarenale AAA zusammengefasst worden. Zwar wurden mittlerweile die perioperativen Ergebnisse bei elektiver Versorgung juxtarenaler AAA hinsichtlich offenem und endovaskulärem Vorgehen dargestellt [16]. Jedoch wurden die Ergebnisse bei EVAR und OAR rein infrarenaler AAA noch nicht ausgewertet. Im Folgenden sollen demnach erstmals die Ergebnisse bei Versorgung juxtarenaler vs. infrarenaler AAA vergleichend berichtet werden.

Im Auftrag des DIGG gGmbH
An dem AAA-Register des DIGG der DGG beteiligten sich in den Jahren 2013-2017 freiwillig jährlich 130 (76-163) gefäßchirurgische Kliniken. Insgesamt wurden 18.629 Patienten erfasst, 17.026 $(91,4 \%)$ mit infrarenalem und 1603 mit juxtarenalem AAA. Bei den infrarenalen AAA wurden $80,5 \%(n=13.707)$ mit EVAR versorgt, bei den juxtarenalen waren es mit 49,0\% ( $n=786)$ signifikant weniger $(p<0,001)$. In die vorliegende Untersuchung wurden nur elektiv versorgte AAA aufgenommen, rupturierte AAA wurden ausgeschlossen. Es handelt sich um eine prospektive Kohortenstudie. Eine externe Validierung der Daten und ein Datenmonitoring waren technisch nicht möglich. Die Definition der Komorbiditäten wurde den behandelnden Kliniken überlassen.

\section{Endovaskuläre Versorgung infrarenale vs. juxtarenale AAA}

Die Patientencharakteristika der Patienten mit infra- und juxtarenalem AAA finden sich in - Tab. 1. Es handelte sich insgesamt um 14.493 Patienten mit AAA, von denen 5,4\% ein juxtarenales AAA aufwiesen. Der Prozentsatz an
Patienten über 80 Jahre war bei juxtarenalen $\mathrm{AAA}$ mit $17,0 \%$ vs. $22,7 \%$ bei infrarenalen AAA signifikant geringer, der Aneurysmadurchmesser hingegen war größer und der Frauenanteil höher. Auch wurden kardiale Begleiterkrankungen bei juxtarenalen AAA häufiger gefunden, umgekehrt war der Diabetes seltener. Insgesamt war präoperativ eine eingeschränkte Nierenfunktion (chronische Niereninsuffizienz Stadium 3-5) bei 1336 (9,7\%) der Patienten mit infrarenalem und bei 99 (12,5\%) Patienten mit juxtarenalem AAA zu beobachten $(p=0,006)$.

Offene Versorgung infrarenale vs. juxtarenale AAA

Die Patientencharakteristika der Patienten mit infra- und juxtarenalem AAA bei OAR finden sich in - Tab. 2. Offen wurden insgesamt 4136 Patienten versorgt, von ihnen hatten $19,8 \%(n=817)$ ein juxtarenales AAA. Bei OAR war der Aneurysmadurchmesser von juxtarenalen AAA signifikant größer als bei den infrarenalen AAA und der Frauenanteil war höher. Kardiale Begleiterkrankungen wurden bei juxtarenalen AAA häufiger gefunden. Hinsichtlich des Diabetes unterschieden sich beide Gruppen nicht. Ei- 
Tab. 1 Infrarenale vs. juxtarenale AAA - Patientencharakteristika und Komorbidität bei EVAR

\begin{tabular}{|c|c|c|c|}
\hline & $\begin{array}{l}\text { Infrarenale AAA } \\
(n=13.707)\end{array}$ & $\begin{array}{l}\text { Juxtarenale AAA } \\
(n=786)\end{array}$ & $p=$ \\
\hline Alter gesamt (Jahre) & $74[72,84]$ & $73[71,58]$ & 0,571 \\
\hline Alter der Männer (Jahre) & $74[72,50]$ & $73[71,36]$ & - \\
\hline Alter der Frauen (Jahre) & $76[75,10]$ & $74[72,70]$ & - \\
\hline Patienten über 80 Jahre, $n$ (\%) & $3109(22,7 \%)$ & $134(17,0 \%)$ & 0,000 \\
\hline Frauen, $n(\%)$ & $1802(13,1 \%)$ & $133(16,9 \%)$ & 0,001 \\
\hline Aneurysmadurchmesser (mm) & $53[54,31]$ & $56[57,80]$ & 0,000 \\
\hline Aneurysmadurchmesser (>65 mm) & $1858(13,7 \%)$ & $179(23,0 \%)$ & 0,000 \\
\hline Hypertoniker, $n(\%)$ & $11.048(89,9 \%)$ & $657(89,9 \%)$ & 0,504 \\
\hline Kardiale Begleiterkrankungen, $n(\%)$ & $6412(46,8 \%)$ & $523(53,8 \%)$ & 0,000 \\
\hline Dyslipoproteinämie, $n(\%)$ & $5919(62,4 \%)$ & $359(57,3 \%)$ & 0,006 \\
\hline Gegenwärtige Raucher, $n$ (\%) & $3582(43,5 \%)$ & $233(41,2 \%)$ & 0,163 \\
\hline COPD, $n(\%)$ & $3931(28,7 \%)$ & $221(28,1 \%)$ & 0,383 \\
\hline Niereninsuffizienz Stadium 3, $n$ (\%) & $1094(8,0 \%)$ & $75(9,5 \%)$ & \multirow[t]{3}{*}{0,02} \\
\hline Niereninsuffizienz Stadium 4, $n$ (\%) & $149(1,1 \%)$ & $15(1,9 \%)$ & \\
\hline Niereninsuffizienz Stadium 5, $n$ (\%) & $93(0,7 \%)$ & $9(1,1 \%)$ & \\
\hline Diabetes mellitus, $n$ (\%) & $2635(34,5 \%)$ & $144(27,3 \%)$ & 0,000 \\
\hline Z. n. Myokardinfarkt, $n$ (\%) & $2259(16,5 \%)$ & $146(18,6 \%)$ & 0,069 \\
\hline Z.n. Schlaganfall, $n$ (\%) & $1320(9,6 \%)$ & $82(10,4 \%)$ & 0,249 \\
\hline
\end{tabular}

Tab. 2 Infrarenale vs. juxtarenale AAA - Patientencharakteristika und Komorbidität bei OAR

\begin{tabular}{|c|c|c|c|}
\hline & $\begin{array}{l}\text { Infrarenale AAA } \\
(n=3319)\end{array}$ & $\begin{array}{l}\text { Juxtarenale AAA } \\
(n=817)\end{array}$ & $p=$ \\
\hline Alter gesamt (Jahre) & $70[68,66]$ & $71[69,34]$ & 0,406 \\
\hline Alter der Männer (Jahre) & $69[68,24]$ & $70[68,88]$ & - \\
\hline Alter der Frauen (Jahre) & $72[70,88]$ & $73[71,32]$ & - \\
\hline Patienten über 80 Jahre, $n(\%)$ & $292(8,8 \%)$ & $81(9,9 \%)$ & 0,176 \\
\hline Frauen, $n(\%)$ & $532(16,0 \%)$ & $152(18,6 \%)$ & 0,043 \\
\hline Aneurysmadurchmesser (mm) & $54[56,33]$ & $56[59,07]$ & 0,000 \\
\hline Aneurysmadurchmesser (>65 mm) & $628(19,2 \%)$ & $191(23,8 \%)$ & 0,002 \\
\hline Hypertoniker, $n(\%)$ & $2717(92,2 \%)$ & $708(95,4 \%)$ & 0,001 \\
\hline Kardiale Begleiterkrankungen, $n(\%)$ & $1149(43,6 \%)$ & $400(49,0 \%)$ & 0,004 \\
\hline Dyslipoproteinämie, $n$ (\%) & $1434(66,5 \%)$ & $401(70,0 \%)$ & 0,065 \\
\hline Gegenwärtige Raucher, $n$ (\%) & $1283(61,9 \%)$ & $302(58,3 \%)$ & 0,072 \\
\hline COPD, $n(\%)$ & $991(29,8 \%)$ & $275(33,7 \%)$ & 0,019 \\
\hline Niereninsuffizienz Stadium 3, $n$ (\%) & $240(7,2 \%)$ & $89(10,9 \%)$ & 0,000 \\
\hline Niereninsuffizienz Stadium 4, $n$ (\%) & $52(1,6 \%)$ & $16(2,0 \%)$ & \\
\hline Niereninsuffizienz Stadium 5, $n$ (\%) & $20(0,6 \%)$ & $12(1,5 \%)$ & \\
\hline Diabetes mellitus, $n(\%)$ & $523(32,8 \%)$ & $139(34,1 \%)$ & 0,329 \\
\hline Z. n. Myokardinfarkt, $n(\%)$ & $502(15,1 \%)$ & $151(18,5 \%)$ & 0,011 \\
\hline Z.n. Schlaganfall, $n$ (\%) & $281(8,5 \%)$ & $94(11,5 \%)$ & 0,005 \\
\hline
\end{tabular}


Gefässchirurgie 2020 25:568-574 https://doi.org/10.1007/s00772-020-00681-w

(c) Der/die Autor(en) 2020

\section{T. Schmitz-Rixen · M. Steffen · D. Böckler · R. T. Grundmann}

\section{Offene und endovaskuläre Versorgung des infrarenalen vs. juxtarenalen Bauchaortenaneurysmas}

\section{Zusammenfassung}

Hintergrund und Zielsetzung. Die vorliegende Untersuchung geht der Frage nach, wie sich die perioperativen Ergebnisse bei elektiver Versorgung juxtarenaler abdomineller Aortenaneurysmen (AAA) von denen bei Versorgung infrarenaler AAA unterscheiden. Material und Methoden. Datenbasis ist das AAA-Register des Deutschen Instituts für Gefäßmedizinische Gesundheitsforschung (DIGG) der Deutschen Gesellschaft für Gefäßchirurgie und Gefäßmedizin (DGG) der Jahre 2013-2017 mit insgesamt 18.629 Patienten, $17.026(91,4 \%)$ mit infrarenalem und 1603 $(8,6 \%)$ mit juxtarenalem AAA. Bei den infrarenalen AAA wurden $80,5 \%(n=13.707)$ endovaskulär (EVAR) und $3319(19,5 \%)$ offen (OAR) versorgt, bei den juxtarenalen waren es 786 Patienten mit EVAR $(49,0 \%)$ und 817 $(51,0 \%)$ mit OAR.
Ergebnisse. Sowohl bei OAR als auch bei EVAR waren Patienten mit juxtarenalen AAA in ihren Ausgangsbedingungen nicht mit Patienten mit infrarenalen AAA vergleichbar. Der Frauenanteil war bei juxtarenalen AAA signifikant höher (bei EVAR 16,9\% vs. $13,1 \%$, bei OAR $18,6 \%$ vs. $16,0 \%$ ) und der Aneurysmadurchmesser war signifikant größer (bei EVAR 57,8 vs. 54,3 mm, bei OAR 59,0 vs. $56,3 \mathrm{~mm}$ ). Patienten mit juxtarenalem AAA zeigten signifikant häufiger als Patienten mit infrarenalem AAA bereits präoperativ eine eingeschränkte Nierenfunktion (12,5\% vs. $9,7 \%$ bei EVAR, $14,4 \%$ vs. $9,4 \%$ bei OAR). Die Klinikletalität betrug bei EVAR 1,0\% (infrarenale AAA) vs. 5,7\% (juxtarenale AAA) $(p=0,000)$, bei OAR $4,2 \%$ (infrarenale AAA) vs. 7,7 \% (juxtarenale AAA) $(p=0,000)$. $\mathrm{Zu}$ einer postoperativen Verschlechterung der Nierenfunktion kam es bei EVAR in 2,0\% der Fälle mit infrarenalen AAA vs. 12,2\% bei juxtarenalen AAA $(p=0,000)$, bei OAR in 7,9\% vs. $19,5 \%(p=0,000)$.

Schlussfolgerungen. Im vorliegenden Krankengut waren die Raten an postoperativen Komplikationen sowie die postoperative Sterblichkeit bei Versorgung juxtarenaler AAA signifikant höher als bei Versorgung infrarenaler AAA, das galt sowohl für EVAR als auch für OAR. Inwieweit dies an den ungünstigeren Ausgangsbedingungen bei Patienten mit juxtarenalen AAA lag und welche Rolle die gewählte Operationstechnik dabei spielte, sollte in weiteren Erhebungen überprüft werden.

\section{Schlüsselwörter}

Register · Endovaskuläre Versorgung · Offene Versorgung · Renale Dysfunktion . Krankenhaussterblichkeit

\section{Open and endovascular repair of infrarenal vs. juxtarenal abdominal aortic aneurysms}

\section{Abstract}

Background and objective. This study investigated the differences in perioperative outcome after elective open repair (OAR) and endovascular repair (EVAR) of juxtarenal and infrarenal abdominal aortic aneurysms (AAA). Material and Methods. Data of 18,629 patients of the AAA registry of the German Institute for Vascular Health Research (DIGG) of the German Society for Vascular Surgery and Vascular Medicine (DGG) treated in 2013-2017 were analyzed, 17,026 patients (91.4\%) with infrarenal and 1603 (8.6\%) with juxtarenal AAA. Of the infrarenal AAA $80.5 \%$ $(n=13,707)$ were treated with EVAR and 3319 (19.5\%) with OAR. In the juxtarenal group 786 patients were treated with EVAR (49.0\%) and 817 (51.0\%) with OAR.
Results. In both OAR and EVAR patients with juxtarenal AAA were not comparable in their initial conditions with patients with infrarenal AAA. With juxtarenal AAA the proportion of women was significantly higher (for EVAR $16.9 \%$ vs. $13.1 \%$, for OAR $18.6 \%$ vs. $16.0 \%$ ) and the aneurysm diameter was significantly larger (for EVAR $57.8 \mathrm{~mm}$ vs. $54.3 \mathrm{~mm}$, for OAR $59.0 \mathrm{~mm}$ vs. $56.3 \mathrm{~mm}$ ). Patients with juxtarenal AAA showed a significantly higher frequency of preoperative renal function impairment than patients with infrarenal AAA (12.5\% vs. $9.7 \%$ for EVAR, $14.4 \%$ vs. $9.4 \%$ for OAR). Hospital mortality with EVAR was $1.0 \%$ (infrarenal AAA) vs. $5.7 \%$ (juxtarenal AAA, $p=0.000$ ), with OAR $4.2 \%$ (infrarenal AAA) vs. $7.7 \%$ (juxtarenal AAA, $p=0.000$ ). Postoperative deterioration of kidney function occurred with EVAR in $2.0 \%$ of patients with infrarenal AAA vs. $12.2 \%$ for juxtarenal AAA $(p=0.000)$, with OAR in $7.9 \%$ vs. $19.5 \%$ $(p=0.000)$.

Conclusions. In the present analysis postoperative complications and postoperative mortality were significantly higher when treating juxtarenal AAA as compared to infrarenal AAA and this was true for EVAR as well as for OAR. It should be clarified in further studies to what extent this was due to less favorable initial conditions for patients with juxtarenal AAA and how the chosen surgical techniques affected the results.

\section{Keywords}

Registries - Endovascular repair - Open repair Renal dysfunction · Hospital Mortality ne eingeschränkte Nierenfunktion (chronische Niereninsuffizienz Stadium 3-5) wurde bei 312 (9,4\%) Patienten mit infrarenalem und bei 117 (14,4\%) Patienten mit juxtarenalem AAA präoperativ gesehen $(p<0,001)$.

\section{Definitionen}

Juxtarenale AAA wurden als solche definiert, wenn bei OAR die Aortenklemme wenigstens oberhalb der ersten Nierenarterie platziert wurde. Bei endovaskulärer Versorgung reichte das Aneurysma bis unterhalb der Nierenarterien und machte primär geplant mindestens einen „scallop“, „fenestration“, „branch“ oder „chimney/snorkel“ in eine Nierenarterie oder Viszeralarterie erforderlich. Eine Verschlechterung der Nierenfunktion wurde angenommen, wenn nach dem Eingriff ein höheres Stadium der Niereninsuffizienz als vor dem Eingriff beobachtet wurde. 


\begin{tabular}{|c|c|c|c|}
\hline & $\begin{array}{l}\text { Infrarenale AAA } \\
(n=13.707)\end{array}$ & $\begin{array}{l}\text { Juxtarenale AAA } \\
(n=786)\end{array}$ & $p=$ \\
\hline Patienten mit postoperativen Komplikationen, $n(\%)$ & $2849(20,7 \%)$ & $244(31,0 \%)$ & 0,000 \\
\hline MACE, $n(\%)$ & $219(1,6 \%)$ & $69(8,8 \%)$ & 0,000 \\
\hline Klinikletalität, $n(\%)$ & $138(1,0 \%)$ & $45(5,7 \%)$ & 0,000 \\
\hline Klinikletalität Männer, $n$ (\%) & $105(0,9 \%)$ & $31(4,7 \%)$ & 0,000 \\
\hline Klinikletalität Frauen, $n(\%)$ & $33(1,8 \%)$ & $14(10,5 \%)$ & 0,000 \\
\hline Klinikletalität Patienten unter 80 Jahre, $n(\%)$ & $82(0,8 \%)$ & $35(5,4 \%)$ & 0,000 \\
\hline Klinikletalität Patienten über 80 Jahre, $n$ (\%) & $56(1,8 \%)$ & $10(7,5 \%)$ & 0,000 \\
\hline $\begin{array}{l}\text { Patienten ohne perioperative Bluttransfusion oder Autotransfu- } \\
\text { sion, } n(\%)\end{array}$ & $11.200(81,8 \%)$ & $548(69,7 \%)$ & 0,000 \\
\hline $\begin{array}{l}\text { Patienten mit Fremdblut, } n(\%) \\
\quad<21 \\
>21\end{array}$ & $\begin{array}{l}675(4,9 \%) \\
-526(3,8 \%) \\
-149(1,1 \%)\end{array}$ & $\begin{array}{l}176(22,4 \%) \\
147(18,7 \%) \\
29(3,7 \%)\end{array}$ & 0,000 \\
\hline
\end{tabular}

\section{Statistik}

Die statistische Auswertung erfolgte mit SPSS 22 (IBM, Armonk, NY, USA). Zur Berechnung der Signifikanzen bei nominalen Variablen wurde der $\chi^{2}$-Test angewandt. Die $p$-Werte entsprechen der Signifikanz aus dem exakten Test nach Fischer. Zur Signifikanzberechnung metrischer Variablen erfolgte ein T-Test. Hierbei wurde jeweils ein Levene-Test auf Varianzgleichheit durchgeführt. Die $p$-Werte wurden hiernach bestimmt. Als Signifikanzniveau wurde $p<0,05$ gewählt.

\section{Ergebnisse}

\section{Endovaskuläre Versorgung infrarenale vs. juxtarenale AAA}

Postoperative Morbidität und Letalität nach EVAR bei infrarenalen und juxtarenalen AAA sind in - Tab. 3 aufgeführt. Postoperative Komplikationen traten bei juxtarenalen AAA signifikant häufiger auf als bei infrarenalen AAA $(31,0 \%$ vs. $20,7 \%, p<0,001)$. MACE („major adverse cardiovascular events $"$ = perioperativer Tod, Schlaganfall oder Herzinfarkt) wurde bei 1,6\% $(n=219)$ der Patienten mit infrarenalem AAA, aber bei 8,8\% $(n=69)$ der Patienten mit juxtarenalem AAA beobachtet $(p<0,001) .81,8 \%$ der Patienten mit infrarenalem AAA kamen ohne perioperative Bluttransfusion oder $\mathrm{Au}$ totransfusion aus, verglichen mit $69,7 \%$ bei juxtarenalem AAA $(p=0,000)$. Zu einer Verschlechterung der Nierenfunktion kam es bei 2,0\% der Patienten mit infrarenalen AAA vs. $12,2 \%$ bei juxtarenalen AAA $(p=0,000$; Odds Ratio 6,7 [95\%-Konfidenzintervall 5,2-8,5]). Eine Dialyse war postoperativ bei $0,9 \%$ vs. $5,6 \%$ erforderlich $(p=0,000)$. Die Klinikletalität betrug 5,7\% (juxtarenale $\mathrm{AAA}$ ) vs. $1,0 \%$ (infrarenale AAA) $(p=0,000)$. Die Klinikletalität war in allen Untergruppen bei juxtarenalen AAA signifikant höher als bei infrarenalen AAA, besonders auffallend war der Unterschied bei den Frauen mit 10,5\% (juxtarenal) vs. 1,8\% (infrarenal).

\section{Offene Versorgung infrarenale vs. juxtarenale AAA}

Postoperative Morbidität und Letalität nach OAR bei infrarenalen und juxtarenalen AAA sind in • Tab. 4 aufgeführt. Postoperative Komplikationen traten bei juxtarenalen AAA signifikant häufiger auf als bei infrarenalen AAA (45,7\% vs. $38,1 \%, p=0,000)$. MACE wurde bei 6,0 \% der Patienten mit infrarenalem AAA und bei $10,3 \%$ der Patienten mit juxtarenalem AAA beobachtet $(p<0,001)$. 33,7\% der Patienten mit infrarenalem AAA kamen ohne perioperative Bluttransfusion oder Autotransfusion aus, verglichen mit $25,2 \%$ bei juxtarenalem AAA $(p=0,000)$. $\mathrm{Zu}$ einer Verschlechterung der Nierenfunktion kam es bei 7,9\% der Patienten mit infrarenalen AAA vs. 19,5\% bei juxtarenalen AAA $(p=0,000)$. Eine Dialyse war postoperativ bei $3,4 \%$ vs. $10,8 \%$ erforderlich $(p=0,000)$. Die Klinikletalität betrug 7,7\% (juxtarenale AAA) vs. $4,2 \%$ (infrarenale AAA). Die Klinikletalität war in allen Untergruppen - außer den über 80-jährigen Patienten - bei juxtarenalen AAA signifikant höher als bei infrarenalen AAA. Besonders auffallend war der Unterschied bei den Frauen mit $11,8 \%$ (juxtarenal) vs. 5,1\% (infrarenal) $(p=0,004)$.

\section{Diskussion}

In einer früheren Analyse hatten wir die Ergebnisse von EVAR und OAR bei Versorgung juxtarenaler AAA miteinander verglichen. Es zeigte sich, dass EVAR im Vergleich zu OAR die signifikant geringere Komplikationsrate aufwies, die postoperative Letalität war jedoch mit 5,7\% vs. $7,7 \%$ nur im Trend niedriger [16]. Wie die vorliegende Untersuchung jetzt ergab, waren juxtarenale AAA sowohl bei EVAR als auch bei OAR mit einer signifikant höheren postoperativen Komplikationsrate, Klinikletalität und MACE im Vergleich zu infrarenalen AAA assoziiert.

Diese Beobachtung, dass juxtarenale AAA signifikant schlechtere Ergebnisse als infrarenale AAA aufweisen, steht im Widerspruch zu einer Erhebung der NSQIP-Datenbank der Jahre 2005 bis 2008, in der Patienten mit offener Versorgung komplexer AAA (AAA mit Einbeziehung der Viszeralarterien ohne Rekonstruktion oder Bypass der Viszeralarterien) sich in ihrer 30-Tage-Letalität (5,7\%) nicht 


\begin{tabular}{|c|c|c|c|}
\hline & $\begin{array}{l}\text { Infrarenale AAA } \\
(n=3319)\end{array}$ & $\begin{array}{l}\text { Juxtarenale AAA } \\
(n=817)\end{array}$ & $p=$ \\
\hline Patienten mit postoperativen Komplikationen, $n$ (\%) & $1261(38,1 \%)$ & $374(45,7 \%)$ & 0,000 \\
\hline $\mathrm{MACE}, n(\%)$ & $199(6,0 \%)$ & $84(10,3 \%)$ & 0,000 \\
\hline Klinikletalität, $n$ (\%) & $139(4,2 \%)$ & $63(7,7 \%)$ & 0,000 \\
\hline Klinikletalität Männer, $n$ (\%) & $112(4,0 \%)$ & $45(6,8 \%)$ & 0,002 \\
\hline Klinikletalität Frauen, $n$ (\%) & $27(5,1 \%)$ & $18(11,8 \%)$ & 0,004 \\
\hline Klinikletalität Patienten unter 80 Jahre, $n(\%)$ & $105(3,5 \%)$ & $54(7,3 \%)$ & 0,000 \\
\hline Klinikletalität Patienten über 80 Jahre, $n(\%)$ & $34(11,6 \%)$ & $9(11,1 \%)$ & 0,537 \\
\hline $\begin{array}{l}\text { Patienten ohne perioperative Bluttransfusion oder Autotransfu- } \\
\text { sion, } n(\%)\end{array}$ & $1118(33,7 \%)$ & $206(25,2 \%)$ & 0,000 \\
\hline $\begin{array}{l}\text { Patienten mit Fremdblut, } n(\%) \\
\quad<21 \\
>21\end{array}$ & $\begin{array}{l}878(26,4 \%) \\
-658(19,8 \%) \\
-220(6,6 \%)\end{array}$ & $\begin{array}{l}261(31,9 \%) \\
180(22,0 \%) \\
81(9,9 \%)\end{array}$ & 0,000 \\
\hline
\end{tabular}

von solchen mit infrarenalen AAA (30Tage-Letalität 5,1\%) unterschieden [13]. In der vorliegenden Studie wurde eine deutlich höhere Klinikletalität bei offener Versorgung juxtarenaler AAA als bei Patel et al. [13] gefunden (7,7\%), während umgekehrt bei infrarenalen AAA die Klinikletalität mit 4,2\% niedriger als bei Patel et al. ausfiel. Eine Erklärung für diese Widersprüche muss offenbleiben, es fällt aber auf, dass in der vorliegenden Untersuchung speziell Frauen bei offener Versorgung juxtarenaler AAA ungünstig abschnitten, mit einer Klinikletalität von $11,8 \%$ vs. $6,8 \%$ bei den Männern $(p=0,043)$. Eine Gender-Differenzierung hatten Patel et al. [13] nicht vorgenommen. Die Ergebnisse unserer Untersuchung stimmen jedoch mit Ergebnissen zur externen Validierung des Risikovorhersagemodells der Vascular Study Group of New England [7] überein. In diesem Modell war weibliches Geschlecht ein signifikanter Prädiktor für ein ungünstigeres Ergebnis bei AAA-Versorgung. Eine suprarenale Position der Aortenklemme im Vergleich zu einer infrarenalen Abklemmung sagte eine 2,3-mal höhere Odds Ratio für die Kliniksterblichkeit nach OAR voraus [7]. Frauen zeigten in der vorliegenden Analyse nach EVAR sowohl bei infrarenalen als auch bei juxtarenalen AAA eine höhere Klinikletalität als Männer, sie waren auch älter, was mit der retrospektiven Analyse der Hospital Episode Statistics (HES) von England von Lowry et al. [10] übereinstimmt.
Liu et al. [9] fanden in einer Metaanalyse (32 Kohortenstudien, 74.969 Patienten) bei Frauen nach endovaskulärer Versorgung infrarenaler AAA eine signifikant höhere 30-Tage-Letalität im Vergleich zu Männern (Odds Ratio 1,67; $95 \%$-KI 1,50-1,87; $p<0,001)$. Auch waren bei Frauen die Raten an weiteren Komplikationen wie Ischämie im Bereich der unteren Extremitäten, an renalen und kardialen Komplikationen nach EVAR höher als bei Männern. Darüber hinaus war das langfristige Überleben bei Frauen reduziert (Hazard Ratio [HR] 1,23; $95 \%$-KI 1,09-1,38; $p=0,001$ ). Als Gründe für die ungünstigeren Ergebnisse nach EVAR bei Frauen im Vergleich zu Männern nannten die Autoren zum einen den relativ größeren AAA-Durchmesser bei Intervention, wenn die Intervention wie bei Männern bei einem Durchmesser von $5,5 \mathrm{~cm}$ indiziert wird (Frauen haben kleinere Aortendurchmesser), zum anderen das höhere Lebensalter der Frauen im Vergleich zu Männern und die ungünstigeren anatomischen Voraussetzungen mit schmaleren Zugangsgefäßen bei endovaskulärer Intervention und eine ungünstigere Aneurysmahalsanatomie. Deery et al. [5] wiesen darauf hin, dass bei Anwendung gleicher Durchmessergrenzen als Operationsindikation Frauen in einem späteren Stadium der AAA-Entwicklung versorgt werden als Männer und schlugen vor, zusätzlich den Aortic Size Index (ASI) zu bestimmen, da adjustiert bei gleichem ASI die Ergebnisunterschiede zwischen Frau- en und Männern nicht mehr signifikant waren. (Der ASI wurde in dieser Erhebung berechnet als Aortendurchmesser/ Körperoberfläche.) Inwieweit demnach der ASI ein besserer Parameter als der Aortendurchmesser speziell bei Frauen für die Indikationsstellung zur Versorgung des AAA wäre, müsste in größeren Erhebungen evaluiert werden.

In der vorliegenden Analyse betrug bei EVAR die Klinikletalität bei infrarenalen AAA 1,0\% und MACE 1,6\% verglichen mit einer Klinikletalität von $5,7 \%$ und MACE 8,8\% bei juxtarenalen AAA. Im Gegensatz hierzu fanden Glebova et al. [8] bei Analyse der American College of Surgeons-National Surgical Quality Improvement Program database bei FEVAR (fenestrierte Endografts) juxtarenaler AAA keine signifikant erhöhte Klinikletalität im Vergleich zu EVAR bei infrarenalen AAA $(2,4 \%$ vs. $1,5 \%)$. In der FEVAR-Gruppe war allerdings wie auch hier die Komplikationsrate höher und der Transfusionsbedarf größer als bei EVAR. Inwieweit die niedrigere Klinikletalität bei FEVAR in dem Kollektiv von Glebova et al. [8] im Vergleich zur vorliegenden Untersuchung auf einem Selektionsbias beruhte, muss offenbleiben. Immerhin wurden bei Glebova et al. nur 2,4\% der Patienten mit FEVAR behandelt, bei 97,7\% der Patienten handelte es sich um solche mit infrarenalem AAA.

In der Datenbank der Vascular Quality Initiative (VQI) der Jahre 2003 bis 2016 identifizierten O’Donnell et al. [11] 
unter 8880 offenen Versorgungen eines AAA 3470 juxtarenale AAA (39\%). Juxtarenale AAA waren wie in der vorliegenden Untersuchung als solche definiert, wenn die Aortenklemme wenigstens oberhalb der ersten Nierenarterie platziert wurde. Das Krankenhausfallvolumen an juxtarenalen AAA wurde in niedrig $(0-<4)$, mittel $(4-14)$ und hoch (>14 Fälle/Jahr) eingeteilt. In dieser Erhebung war ein geringes Fallvolumen an juxtarenalen AAA mit einer höheren Kliniksterblichkeit assoziiert, mit einer Klinikletalität von $9,0 \%, 4,9 \%$ und 3,9\%. Diese Assoziation war unabhängig davon, wie häufig ein Krankenhaus generell offene Eingriffe bei AAA durchführte, was bedeutet, dass es spezifisch auf die Fallzahl an juxtarenalen AAA ankam. Die Autoren forderten, komplexe AAA an erfahrene Zentren zu überweisen. Die Klinikletalität von $7,7 \%$ in der Gruppe der offen operierten juxtarenalen AAA in der vorliegenden Untersuchung unterstützt diese Forderung: $27,7 \%$ aller OAR bei juxtarenalem AAA wurden in unserer Untersuchung in Krankenhäusern mit $<5$ juxtarenalen AAA/Jahr vorgenommen.

Inwieweit unsere Ergebnisse für Deutschland repräsentativ sind, ist zweifelhaft, zumindest gilt dies für die Versorgung der juxtarenalen AAA. Gerade große Zentren waren in unserer Datenerhebung unterrepräsentiert, mit nur 12 von 37 Universitätskliniken. So wurde von Silveira et al. [15] bei endovaskulärer Versorgung juxtarenaler AAA von 35 achtzigjährigen Hochrisikopatienten, die für OAR nicht geeignet waren, lediglich eine 30-Tage-Letalität von 2,9\% (1 Todesfall) und eine frühe Morbidität von $14,3 \%$ berichtet. In einem italienischen Zentrum machte die 30-TageLetalität bei OAR von 155 juxtarenalen AAA sogar nur $0,6 \%$ aus, die postoperative Morbidität $10 \%$ [6]. Fünf akademische Zentren aus Frankreich berichteten über eine multizentrische konsekutive Serie von 315 OAR juxtarenaler AAA [3]. In dieser Serie wurden lediglich 3 (0,9\%) Todesfälle postoperativ (30-Tage-Letalität und Klinikletalität eingeschlossen) gesehen, postoperative Komplikationen traten in 14,6\% auf. Auch fällt in unserer Untersuchung der geringe Anteil an juxtarenalen AAA am Gesamtkrankengut bei EVAR auf $(5,4 \%)$ verglichen mit immerhin $19,8 \%$ bei OAR $(p<0,001)$. Ein Selektionsbias bei der Krankenhausauswahl lässt sich demnach für die vorliegende, aber auch für die anderen Untersuchungen nicht ausschließen. Dies gilt generell für relativ kleine Kollektive. Deery et al. [4] berichteten über 443 offene Versorgungen bei komplexen AAA, definiert als Versorgungen, bei denen die Klemme entweder suprarenal (einschließlich zwischen den Nierenarterien) oder supracoeliakal positioniert wurde vs. 1041 OAR bei infrarenalen AAA. Es handelte sich um das Krankengut der Vascular Study Group of New England der Jahre 2003 bis 2011 von lediglich 14 Krankenhäusern. In diesem Kollektiv machten komplexe AAA am Gesamtkrankengut der AAA 28,9\% aus, mit einer 30-Tage-Letalität von 1,2\% bei infrarenalen AAA vs. $2,7 \%$ bei suprarenalem Clamping oder Clamping oberhalb einer Nierenarterie.

In der vorliegenden Untersuchung waren sowohl bei OAR als auch bei EVAR Patienten mit juxtarenalen AAA in ihren Ausgangsbedingungen nicht mit Patienten mit infrarenalen AAA vergleichbar, was sich negativ auf die Ergebnisse bei juxtarenalen AAA ausgewirkt haben könnte. Bei den juxtarenalen AAA war der Frauenanteil im Vergleich zu den Männern relativ höher und der Aneurysmadurchmesser signifikant größer (- Tab. 1 und 2). Ebenso bedeutsam war, dass bei EVAR und bei OAR Patienten mit juxtarenalem AAA signifikant häufiger als Patienten mit infrarenalem AAA bereits präoperativ eine eingeschränkte Nierenfunktion aufwiesen. O'Donnell et al. [12] haben gezeigt, dass bei offener Versorgung juxtarenaler AAA Patienten mit präoperativ eingeschränkter Nierenfunktion (GFR $<60 \mathrm{ml} / \mathrm{min} / 1,73 \mathrm{~m}^{2}$ ) postoperativ ein signifikant höheres Risiko einer akuten Nierenschädigung aufweisen als Patienten mit einer GFR $>60 \mathrm{ml} / \mathrm{min} / 1,73 \mathrm{~m}^{2}$, was sich negativ auf die perioperative Sterblichkeit und Langzeitsterblichkeit auswirkte. In der vorliegenden Untersuchung wurde dementsprechend bei juxtarenalem AAA signifikant häufiger eine postoperative Verschlechterung der Nierenfunktion gefunden als bei infra- renalem AAA, bei EVAR in $12,2 \%$ vs. $2,0 \%(p=0,000)$, bei OAR in $19,5 \%$ vs. $7,9 \%(p=0,000)$.

Verglichen mit unseren bisherigen Jahresberichten, die juxta- und infrarenale AAA - wie übrigens fast alle Register - stets zusammengefasst haben, machte die Klinikletalität der rein infrarenalen AAA bei EVAR jetzt 1,0\% (früher 1,26\%), bei OAR 4,2\% (früher $4,9 \%)$ aus. Speziell für OAR ist diese Korrektur von Bedeutung, da sie belegt, dass wir über alle Jahre zumindest bei infrarenalen AAA deutlich unter dem Schwellenwert einer Klinikletalität von $5,0 \%$ geblieben sind, der laut Leitlinien der Society for Vascular Surgery von einer Klinik nicht überschritten werden sollte, die AAA offen behandelt [2]. Gleichwohl besteht aber speziell bei OAR weiterhin ein Verbesserungspotenzial, verglichen mit den Daten einer Metaanalyse von 51 Studien (189.022 Patienten) [1], in der nach EVAR eine gepoolte 30 -Tage-Letalität von $1,16 \%$ (95\%-KI $0,92$ bis 1,39$)$, nach OAR von $3,27 \%$ (95\%-KI 2,71 bis 3,83) berechnet wurde (alle AAA zusammengefasst, infra- und juxtarenal nicht unterschieden). Dass solche Zielwerte auch in flächendeckenden Registern zu erreichen sind, zeigt der Jahresbericht 2019 der Vascular Society of Great Britain and Ireland [17] zur Versorgung des intakten AAA (ebenfalls juxta- und infrarenale AAA zusammengefasst): dort wurde für die 3-JahresPeriode 2016 bis 2018 bei EVAR eine Klinikletalität von lediglich $0,5 \%$ und für OAR von 3,1\% genannt! Gleichwohl ziehen wir aus der vorliegenden Analyse die Konsequenz, aus Vergleichsgründen weiterhin in künftigen Jahresberichten die Komplikationsraten bei Versorgung des AAA (zusammengefasst) anzugeben und zusätzlich zwischen infra- und juxtarenalen AAA zu differenzieren.

Zusammenfassend waren in der vorliegenden Untersuchung die Ergebnisse bei Versorgung juxtarenaler AAA signifikant schlechter als bei Versorgung infrarenaler AAA, dies galt sowohl für EVAR als auch für OAR. Die große Diskrepanz deckt sich nur bedingt mit anderen Registererhebungen. Speziell die hohe Klinikletalität bei den Frauen, sowohl nach EVAR als auch nach OAR, wurde 
in diesem Ausmaß bei Versorgung juxtarenaler AAA bisher nicht berichtet. Ein Selektionsbias ist nicht auszuschließen. Wesentliches Ziel muss es folglich sein, mehr Zentren als bisher zur Teilnahme an dem DIGG-Register zu motivieren, damit aussagekräftige Daten zur Versorgungssituation speziell juxtarenaler AAA in Deutschland gewonnen werden können.

\section{Korrespondenzadresse}

\section{Prof. Dr. R. T. Grundmann}

Universitäres Herz- und Gefäßzentrum UKE Hamburg, Universitätsklinikum HamburgEppendorf

Martinistr. 52, 20246 Hamburg, Deutschland r.grundmann@uke.de

Danksagung. Unser Dank gilt allen Kliniken, die sich über die Jahre an dem AAA-Register beteiligt haben.

Funding. Open Access funding provided by Projekt DEAL.

\section{Einhaltung ethischer Richtlinien}

Interessenkonflikt. T. Schmitz-Rixen, M. Steffen, D. Böckler und R.T. Grundmann geben an, dass kein Interessenkonflikt besteht.

Für diesen Beitrag wurden von den Autoren keine Studien an Menschen oder Tieren durchgeführt. Für die aufgeführten Studien gelten die jeweils dort angegebenen ethischen Richtlinien.

Open Access Dieser Artikel wird unter der Creative Commons Namensnennung 4.0 International Lizenz veröffentlicht, welche die Nutzung, Vervielfältigung, Bearbeitung, Verbreitung und Wiedergabe in jeglichem Medium und Format erlaubt, sofern Sie den/die ursprünglichen Autor(en) und die Quelle ordnungsgemäß nennen, einen Link zur Creative Commons Lizenz beifügen und angeben, ob Änderungen vorgenommen wurden.

Die in diesem Artikel enthaltenen Bilder und sonstiges Drittmaterial unterliegen ebenfalls der genannten Creative Commons Lizenz, sofern sich aus der Abbildungslegende nichts anderes ergibt. Sofern das betreffende Material nicht unter der genannten Creative Commons Lizenz steht und die betreffende Handlung nicht nach gesetzlichen Vorschriften erlaubt ist, ist für die oben aufgeführten Weiterverwendungen des Materials die Einwilligung des jeweiligen Rechteinhabers einzuholen.

Weitere Details zur Lizenz entnehmen Sie bitte der Lizenzinformation auf http://creativecommons.org/ licenses/by/4.0/deed.de.

\section{Literatur}

1. Bulder RMA, Bastiaannet E, Hamming JF, Lindeman JHN (2019) Meta-analysis of long-term survival after elective endovascular or open repair of abdominal aortic aneurysm. Br J Surg 106:523-533

2. ChaikofEL, Dalman R, Eskandari MKet al (2018) The Society for Vascular Surgery practice guidelines on the care of patients with an abdominal aortic aneurysm. JVasc Surg 67:2-77

3. Chaufour X, Segal J, Soler R et al (2020) Editor's choice-durability of open repair of juxtarenal abdominal aortic aneurysms: a multicentre retrospective study in five French academic centres. Eur JVasc Endovasc Surg 59:40-49

4. Deery SE, Lancaster RT, Baril DT et al (2016) Contemporary outcomes of open complex abdominal aortic aneurysm repair. J Vasc Surg 63:1195-1200

5. Deery SE, Soden PA, Zettervall SL et al (2017) Sex differences in mortality and morbidity following repair of intact abdominal aortic aneurysms. J Vasc Surg 65:1006-1013

6. Desole A, Ferrari A, Tosato F, Milite D (2019) Open repair for juxtarenal aortic aneurysm: short and long-term results. Ann Vasc Surg 54:161-165

7. Eslami MH, Rybin DV, Doros G, Siracuse JJ, Farber A (2018) External validation of Vascular Study Group of New England risk predictive model of mortality after elective abdominal aorta aneurysm repair in the Vascular Quality Initiative and comparison against established models. JVasc Surg 67:143-150

8. Glebova NO, Selvarajah S, Orion KC et al (2015) Fenestrated endovascular repair of abdominal aortic aneurysms is associated with increased morbidity but comparable mortality with infrarenal endovascular aneurysm repair. J Vasc Surg 61:604-610

9. Liu Y, Yang Y, Zhao J et al (2020) Systematic review and meta-analysis of sex differences in outcomes after endovascular aneurysm repair for infrarenal abdominal aortic aneurysm. J Vasc Surg 71:283-296.e4

10. Lowry D, Singh J, Mytton J, Tiwari A (2016) Sexrelated outcome inequalities in endovascular aneurysm repair. Eur J Vasc Endovasc Surg 52:518-525

11. O'Donnell TFX, Boitano LT, Deery SE et al (2020) Hospital volume matters: the volume-outcome relationship in open juxtarenal AAA repair. Ann Surg 271:184-190

12. O'Donnell TFX, Boitano LT, Deery SE et al (2019) Factors associated with postoperative renal dysfunction and the subsequent impact on survival after open juxtarenal abdominal aortic aneurysm repair. JVasc Surg 69:1421-1428

13. Patel VI, Lancaster RT, Conrad MF et al (2011) Comparable mortality with open repair of complex and infrarenal aortic aneurysm. J Vasc Surg 54:952-959

14. Schmitz-Rixen T, Steffen M, Böckler D, Grundmann RT (2020) Versorgung des abdominellen Aortenaneurysmas (AAA) 2018. Register-Bericht des DIGG derDGG. Gefässchirurgie 25:117-123

15. Silveira D, Pitoulias G, Torsello G, Donas KP (2016) Outcomes of total endovascular treatment of juxtarenal aortic aneurysms in octogenarians. JVasc Surg 63:909-914

16. Steffen M, Schmitz-Rixen T, Böckler D, Grundmann RT, DIGG gGmbH (2020) Comparison of open and endovascular repair of juxtarenal abdo- minal aortic aneurysms. Langenbecks Arch Surg 405:207-213

17. National Vascular Registry, Waton $S$, Johal A Heikkila K et al (2019) Annual report. London: the royal college of surgeons of england, november 2019. https://www.vsqip.org.uk/content/ uploads/2019/12/NVR-2019-Annual-Report.pdf. Zugegriffen: Juni 2020 